\title{
Case Report \\ Successful Treatment of Disseminated Subcutaneous Panniculitis-Like T-Cell Lymphoma with Single Agent Oral Cyclosporine as a First Line Therapy
}

\author{
Nida Iqbal and Vinod Raina \\ Department of Medical Oncology, Dr. B. R. A. Institute Rotary Cancer Hospital, All India Institute of Medical Sciences, \\ New Delhi 110029, India \\ Correspondence should be addressed to Nida Iqbal; nida.iqbal55@yahoo.com
}

Received 6 July 2014; Accepted 16 November 2014; Published 23 November 2014

Academic Editor: Alireza Firooz

Copyright (C) 2014 N. Iqbal and V. Raina. This is an open access article distributed under the Creative Commons Attribution License, which permits unrestricted use, distribution, and reproduction in any medium, provided the original work is properly cited.

\begin{abstract}
Subcutaneous panniculitis-like T-cell lymphoma (SPTL) is a rare cutaneous neoplasm of mature cytotoxic T-cells. Currently there are no standardized therapies for SPTL; however good responses have been seen with chemotherapy regimens generally employed for B-cell lymphomas. Cyclosporine, an immunosuppressant, has shown good responses in relapsed/refractory SPTL; however its use in first line setting is not well established. We, herein, describe a 22-year-old girl with disseminated SPTL who attained complete clinical remission with single agent oral cyclosporine used as a first line therapy.
\end{abstract}

\section{Introduction}

Subcutaneous panniculitis-like T-cell lymphoma (SPTL) is a type of T-cell lymphoma with clinicopathologic features simulating panniculitis and associated with an aggressive clinical course [1]. There are two subtypes, TCR alpha/beta and TCR gamma/delta [2]. While TCR alpha/beta generally have a CD4-, CD8+, CD56- phenotype and a favorable prognosis, TCR gamma/delta typically have a CD4-, CD8T-cell phenotype with frequent coexpression of CD56 and a poor prognosis [3]. SPTL most commonly affects patients in 4 th decade of life with a female predominance [4]. Patients present with subcutaneous nodules or plaques most commonly on extremities and trunk that, on pathologic evaluation, demonstrate cellular infiltrates in the subcutaneous fat, generally with sparing of the overlying epidermis. Metastatic disease is very rare. Clinical and systemic symptoms are nonspecific. There is no standardized treatment for SPTL [4]. CHOP-like chemotherapy is generally used as an initial therapy. Cyclosporine, an immunosuppressant, has shown activity in relapsed/refractory SPTL $[5,6]$. However, it is still not clear whether cyclosporine as a single agent is able to induce remissions in patients with disseminated SPTL and the question of its use as first line therapy remains unanswered.

In this report, we describe complete clinical remission of disseminated SPTL (TCR alpha/beta) in a 22-year-old girl with single agent oral cyclosporine used as a first line therapy.

\section{Case Report}

A 22-year-old girl presented with the complaints of intermittent fever, decreased appetite, and progressively increasing nodular swellings over both cheeks, lower back, both gluteal areas, and lower limbs. On physical examination, the patient was febrile with ECOG performance status of 2 . There was mild hepatosplenomegaly with no peripheral lymphadenopathy. Skin examination revealed nodular swelling 3-4 cm over both cheeks and $2-3 \mathrm{~cm}$ swellings over lower back, both gluteal areas, and right thigh. There was an ulcerative lesion approximately $3 \mathrm{~cm}$ over left calf with surrounding hyperpigmented area (Figure 1(a)). The laboratory investigations revealed haemoglobin level of $8.7 \mathrm{~g} / \mathrm{dL}$, white blood cell count of $4.1 \times 10^{9} / \mathrm{L}$, platelet count of $275 \times 10^{9} / \mathrm{L}$, serum $\mathrm{LDH}$ of $228 \mathrm{U} / \mathrm{L}$, and normal renal and liver function tests. 


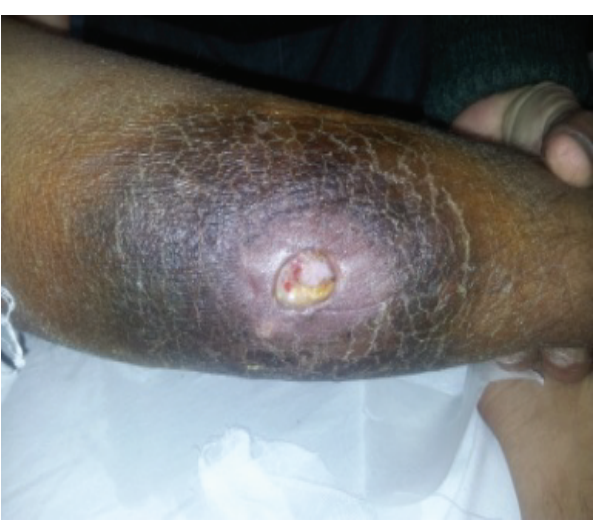

(a)

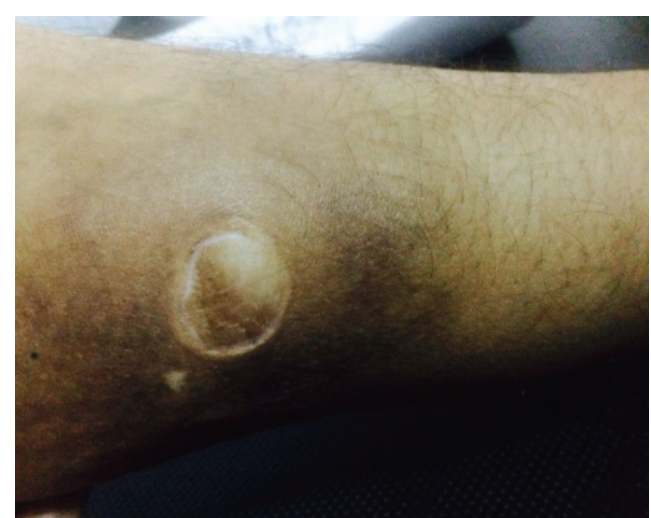

(b)

Figure 1: (a) Ulcerative lesion with surrounding hyperpigmented area over left calf before treatment. (b) Healed lesion 18 months after completion of therapy.

The serologic tests for human immunodeficiency virus and hepatitis B and C were negative. Contrast enhanced CT chest and abdomen did not reveal any abnormality. PET scan revealed diffuse uptake in subcutaneous fat, left breast, liver, and spleen. Bone marrow examination was normal. There was no evidence of hemophagocytic syndrome. An excisional biopsy from the skin nodule showed septal and interstitial panniculitis with fat necrosis and atypical lymphoid cell infiltrate focally lining the adipocytes, mainly limited to subcutaneous tissue and lower dermis. Foamy histiocytes with beanbag cells were also identified in the interstitium. Immunohistochemistry analysis showed that tumor cells expressed CD3 and CD8 but were negative for CD20, CD4, and $\mathrm{CD} 56 . \mathrm{Ki}-67$ was not measured. These findings were consistent with the diagnosis of subcutaneous panniculitislike T-cell lymphoma (alpha/beta subtype).

She was started on oral cyclosporine $4 \mathrm{mg} / \mathrm{kg} / \mathrm{d}$ as a single agent. Patient responded well with resolution of all systemic manifestations of disease within two weeks of initiation of therapy. All skin nodules resolved completely within two months. She was continued on same dose of cyclosporine for next 5 months and finally the drug was tapered over 1 month. She tolerated the therapy well except for mild hirsutism. PET scan done at 6 months of treatment showed complete resolution of all subcutaneous nodules with mild uptake in left calf. Currently, the patient is in clinical remission 18 months after completion of therapy (Figure 1(b)).

\section{Discussion}

Subcutaneous panniculitis-like T-cell lymphoma (SPTL) was first described by Gonzalez et al. in 1991 [1] and defined as a distinct entity by the World Health Organization (WHO) classification in $2001[7,8]$. SPTL is a cutaneous condition and metastatic disease or visceral involvement is uncommon. CD56 is an important marker in T-cell lymphomas as CD56 positive tumors have worse outcome in view of disseminated disease and hemophagocytosis [9]. Only cases with TCR gamma/delta phenotype (mostly CD56 positive) tend to have metastasis to various organs, including lungs, liver, kidneys, and the central nervous system. Hemophagocytic syndrome (HPS) characterized by fever, pancytopenia, hepatosplenomegaly, and coagulopathy is most commonly seen in TCR gamma/delta phenotype and associated with aggressive outcome [10]. TCR alpha/beta phenotype is generally CD56 negative and associated with favourable outcome. The overall five-year survival rate for TCR alpha/beta exceeds $80 \%$; however, in the presence of HPS, it reduces to less than $50 \%$. In cases of TCR gamma/delta, the five-year survival rate is less than $20 \%$ in either group [4].

Due to rarity of disease, no standardized therapy for SPTL currently exists. For indolent local disease, local radiotherapy can be used as an effective treatment modality. For indolent disease with a more generalized distribution, systemic biologic agents may be used, such as bexarotene and interferon, as well as low-dose chemotherapy with agents such as methotrexate. For aggressive presentations, doxorubicinbased therapies are most commonly used, with overall complete or partial remission rates of 50\%. Fludarabine-based chemotherapies have shown an overall remission rates of more than $70 \%$ in a few case reports $[11,12]$. A case of SPTL with HPS resistant to CHOP regimen achieved complete remission after combination chemotherapy using BFM-90 protocol [11]. High-dose chemotherapy followed by stem cell transplantation have been reported to produce the highest response rates $[13,14]$.

Immunosuppressive therapy with steroids and cyclosporine have shown good results in the treatment of relapsed/ refractory SPTL in a few case reports $[5,6]$. Cyclosporine, a calcineurin inhibitor, is a potent immunosuppressant. The mechanism of action of cyclosporine in SPTL is downregulation of cytokines. Despite its magical effects in a few cases of relapsed/refractory SPTL, we could not find any report of its use in upfront setting. Our case is the first of its kind in which upfront use of cyclosporine was able to maintain durable remission in patient with disseminated SPTL. 


\section{Conclusion}

Because of lack of standard treatment to SPTL, cyclosporine may be a good option as a first line therapy even in patients with disseminated disease due to lack of side effects and ease of administration. However, its benefit in patients with TCR gamma/delta phenotype needs to be confirmed by further studies.

\section{Conflict of Interests}

The authors declare that there is no conflict of interests regarding the publication of this paper.

\section{References}

[1] C. L. Gonzalez, L. J. Medeiros, R. M. Braziel, and E. S. Jaffe, "T-cell lymphoma involving subcutaneous tissue: a clinicopathologic entity commonly associated with hemophagocytic syndrome," The American Journal of Surgical Pathology, vol. 15, no. 1, pp. 17-27, 1991.

[2] K. E. Salhany, W. R. Macon, J. K. Choi et al., "Subcutaneous panniculitis-like T-cell lymphoma: clinicopathologic, immunophenotypic, and genotypic analysis of alpha/beta and gamma/delta subtypes," The American Journal of Surgical Pathology, vol. 22, no. 7, pp. 881-893, 1998.

[3] C. Massone, A. Chott, D. Metze et al., "Subcutaneous, blastic natural killer (NK), NK/T-cell, and other cytotoxic lymphomas of the skin: a morphologic, immunophenotypic, and molecular study of 50 patients," The American Journal of Surgical Pathology, vol. 28, no. 6, pp. 719-735, 2004.

[4] R. Willemze, P. M. Jansen, L. Cerroni et al., "Subcutaneous panniculitis-like T-cell lymphoma: definition, classification, and prognostic factors: an EORTC Cutaneous Lymphoma Group Study of 83 cases," Blood, vol. 111, no. 2, pp. 838-845, 2008.

[5] P. Rojnuckarin, T. N. Nakorn, T. Assanasen, P. Wannakrairot, and T. Intragumtornchai, "Cyclosporin in subcutaneous panniculitis-like T-cell lymphoma," Leukemia \& Lymphoma, vol. 48 , no. 3, pp. 560-563, 2007.

[6] S. I. Go, W. S. Lee, M. H. Kang, D. C. Kim, J. H. Lee, and I. S. Kim, "Cyclosporine a treatment for relapsed subcutaneous panniculitis-like T-cell lymphoma: a case with long-term follow-up," Korean Journal of Hematology, vol. 47, no. 2, pp. 146149, 2012.

[7] E. S. Jaffe, N. L. Harris, H. Stein, and J. W. Vardiman, Eds., Pathology and Genetics of Tumours of Haematopoietic and Lymphoid Tissues, World Health Organization Classification of Tumours, IARC Press, Lyon, France, 2001.

[8] R. Willemze, E. S. Jaffe, G. Burg et al., "WHO-EORTC classification for cutaneous lymphomas," Blood, vol. 105, no. 10, pp. 3768-3785, 2005.

[9] M. Takeshita, S. Okamura, Y. Oshiro et al., "Clinicopathologic differences between 22 cases of CD56-negative and CD56positive subcutaneous panniculitis -like lymphoma in Japan," Human Pathology, vol. 35, no. 2, pp. 231-239, 2004.

[10] R. S. Go and S. M. Wester, "Immunophenotypic and molecular features, clinical outcomes, treatments, and prognostic factors associated with subcutaneous panniculitis-like T-cell lymphoma: a systematic analysis of 156 patients reported in the literature," Cancer, vol. 101, no. 6, pp. 1404-1413, 2004.
[11] C.-S. Chim, F. Loong, W.-K. Ng, and Y.-L. Kwong, "Use of fludarabine-containing chemotherapeutic regimen results in durable complete remission of subcutaneous panniculitis-like T-cell lymphoma," American Journal of Clinical Dermatology, vol. 9, no. 6, pp. 396-398, 2008.

[12] K. Medhi, R. Kumar, A. Rishi, L. Kumar, and S. Bakhshi, "Subcutaneous panniculitislike T-cell lymphoma with hemophagocytosis: complete remission with BFM-90 protocol," Journal of Pediatric Hematology/Oncology, vol. 30, no. 7, pp. 558-561, 2008.

[13] K. Koizumi, K. Sawada, M. Nishio et al., "Effective high-dose chemotherapy followed by autologous peripheral blood stem cell transplantation in a patient with the aggressive form of cytophagic histiocytic panniculitis," Bone Marrow Transplantation, vol. 20, no. 2, pp. 171-173, 1997.

[14] P. Reimer, T. Rüdiger, J. Müller, C. Rose, M. Wilhelm, and F. Weissinger, "Subcutaneous panniculitis-like T-cell lymphoma during pregnancy with successful autologous stem cell transplantation," Annals of Hematology, vol. 82, no. 5, pp. 305-309, 2003. 


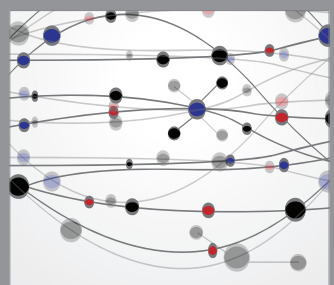

The Scientific World Journal
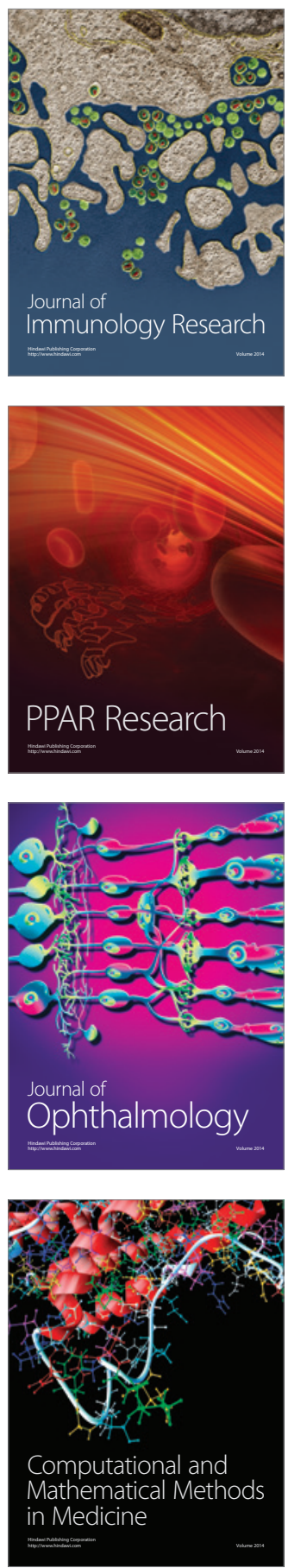

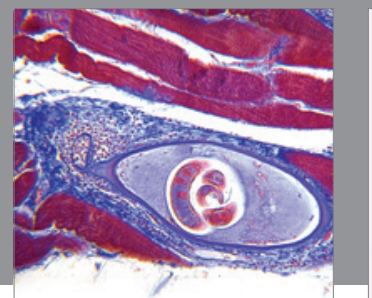

Gastroenterology

Research and Practice
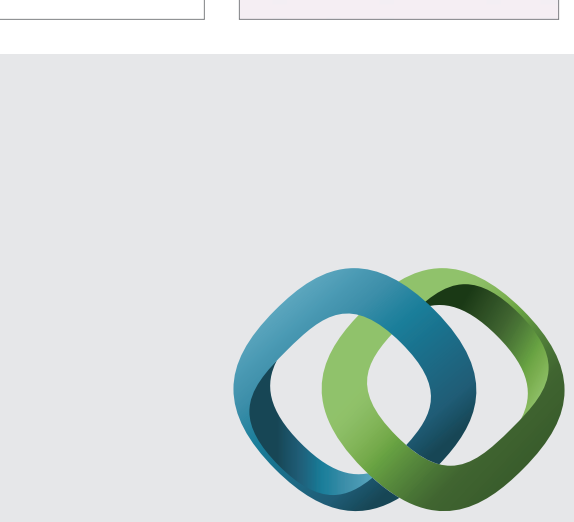

\section{Hindawi}

Submit your manuscripts at

http://www.hindawi.com
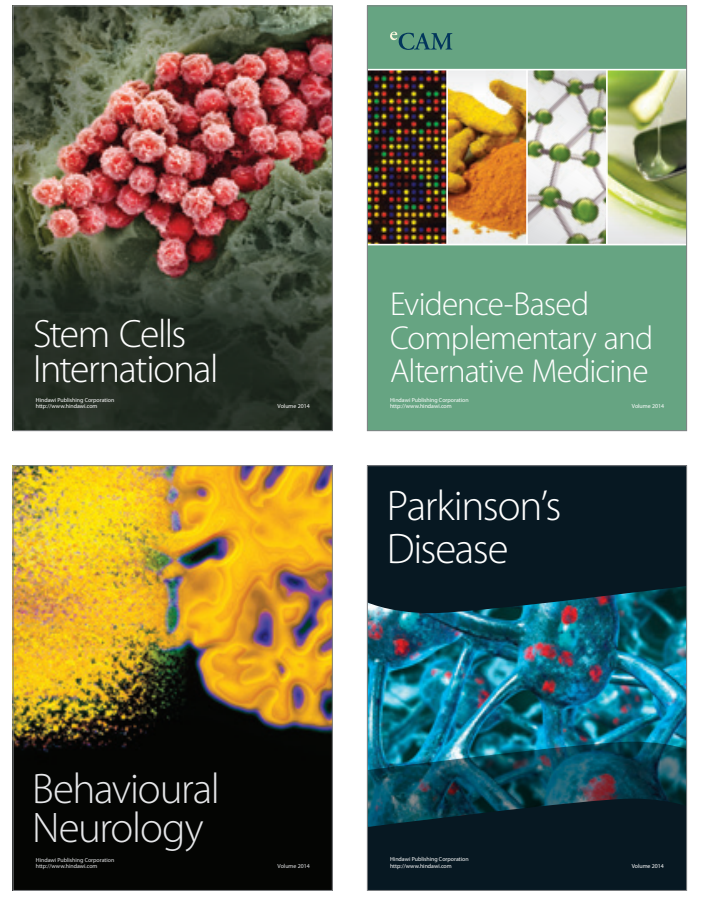
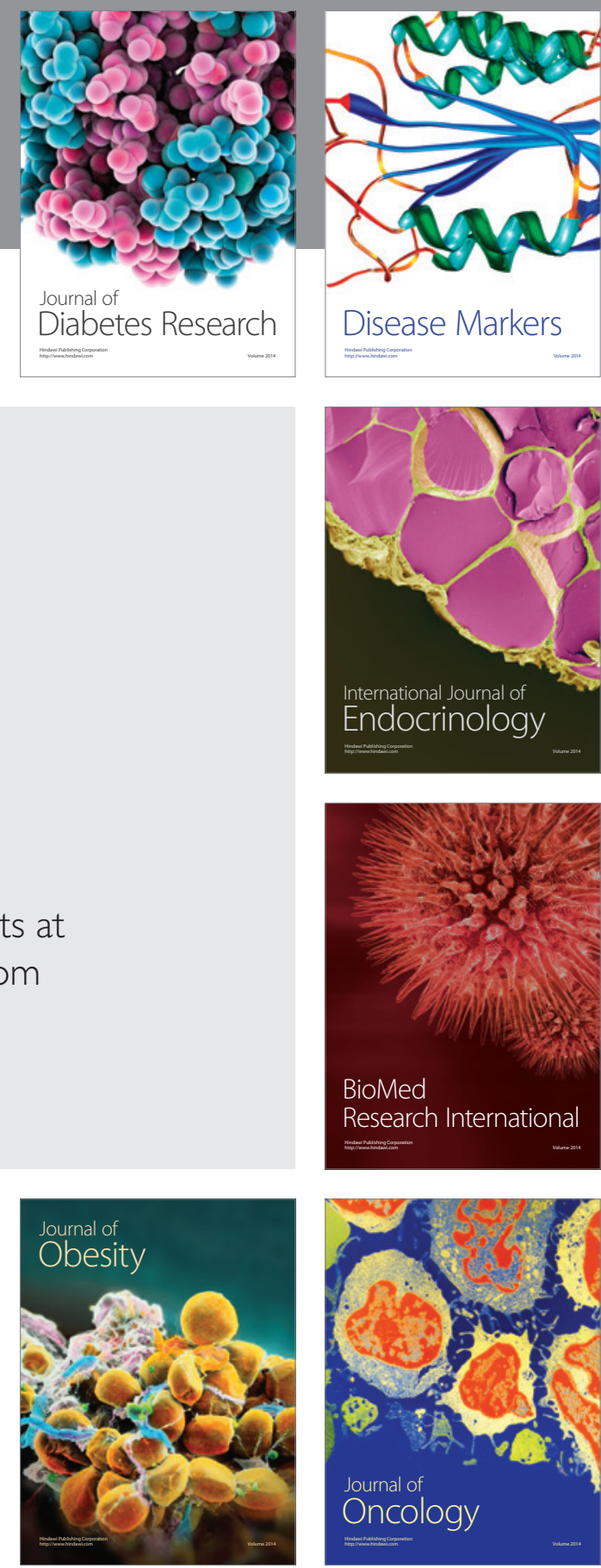

Disease Markers
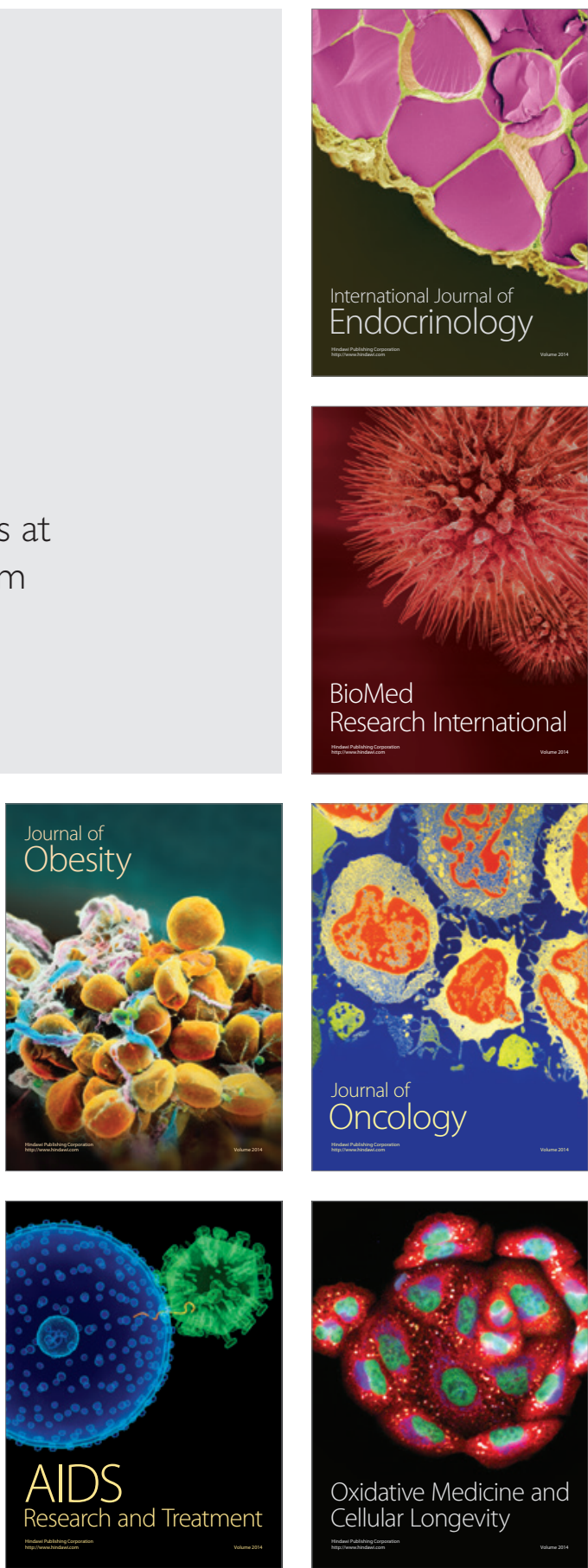